INJE-TP-00-2, hep-th/0001211

\title{
Schwarzschild black hole in the dilatonic domain wall
}

\author{
Y.S. Myung and H.W. Lee \\ Department of Physics, Inje University, Kimhae 621-749, Korea
}

\begin{abstract}
In the dilatonic domain wall model, we study the Schwarzschild black hole as a solution to the Kaluza-Klein (KK) zero mode effective action which is equivalent to the Brans-Dicke (BD) model with a potential. This can describe the large Randall-Sundrum (RS) black hole whose horizon is to be the intersection of the black cigar with the brane. The black cigar located far from the $\mathrm{AdS}_{5}$-horizon is known to be stable, but any explicit calculation for stability of the RS black hole at $z=0$ is not yet performed. Here its stability is investigated against the $z$-independent perturbations composed of odd, even parities of graviton $\left(h_{\mu \nu}\right)$ and $\mathrm{BD} \operatorname{scalar}\left(h_{44}=2 \varphi\right)$. It seems that the RS black hole is classically unstable because it has a potential instability at wavelength with $\lambda>1 /(2 k)$. However, this is not allowed inside an $\mathrm{AdS}_{5}$-box of the size with $1 /(2 k)$. Thus the RS black hole becomes stable. The RS black hole can be considered as a stable remnant at $z=0$ of the black cigar.
\end{abstract}




\section{INTRODUCTION}

Recently there has been much interest in the Randall-Sundrum brane world [1-3]. A key idea of this model is that our universe may be a brane embedded in the higher dimensional space. A concrete model is a single 3-brane embedded in the five-dimensional anti-de Sitter space $\left(\mathrm{AdS}_{5}\right)$, which acts like a box of the size $\sim 1 / k$. Randall and Sundrum have shown that a longitudinal part $\left(h_{\mu \nu}\right)$ of the metric fluctuations satisfies the Schrödinger-like equation with an attractive delta-function. As a result, the massless KK modes which describe the localized gravity on the brane were found. Furthermore, the massive KK modes lead to corrections to the Newtonian potential like as $V(r)=G_{N} \frac{m_{1} m_{2}}{r}\left(1+\frac{1}{k^{2} r^{2}}\right)$.

However, we would like to point out that this has been done in the 4D Minkowski space with the RS gauget. It seems that this gauge is so restrictive. In order to have welldefined theory on the brane, one has to include non-zero transverse parts of $h_{4 \mu}$ and $h_{44}$ at the beginning. Ivanov and Volovich discussed along this direction by choosing the 5D de Donder gauge [4,5]. Also authors in Ref. [6] studied the propagation of the metric including with non-zero transverse parts. It turned out that there are no massless scalar and vector propagations on the RS background. This implies that the RS gauge is a good choice for describing the brane world. Furthermore, it is shown that the RS Minkowski spacetime is stable only under the RS gauge.

If the Minkowski metric on the brane is replaced by any 4D Ricci-flat one, for example the Schwarzschild metric, the 5D metric still satisfies the Einstein equation with a negative cosmological constant. In this process we obtain a black string solution in an $\mathrm{AdS}_{5}$ [ [7]. This is given by $\bar{G}_{M N}^{\mathrm{BS}}=H^{-2}(z)\left[\bar{g}_{\mu \nu}^{S}, 1\right]$ with the Schwarzschild metric $\bar{g}_{\mu \nu}^{S}=\operatorname{diag}[-(1-$ $\left.2 M / r),(1-2 M / r)^{-1}, r^{2}, r^{2} \sin ^{2} \theta\right]$. It is shown that this black string is unstable near the $\mathrm{AdS}_{5}$ horizon of $z=\infty$ but it is stable far from the horizon. This is a result of the GregoryLaflamme instability [8], which states that the black string has a tendency to fragment near the $\mathrm{AdS}_{5}$-horizon [9]. A stable object left behind would resemble a black cigar, although we do not know its explicit metric. Hence we have the RS black hole-picture on the brane located at $z=0$. The horizon of black hole on the brane will be determined from the intersection of the black cigar with the brane. Here we assume that this is large such as $r_{E H}=2 M>1 / k$. In this case an observer on the brane perceives exactly the Schwarzschild solution, without any correction arising from the extra dimension [10]. In other words, any massive KK mode are not excited in this circumstances. Then the zero modes of the bulk graviton can describe this situation very well. Hence the zero mode approach becomes a powerful technique in the study of black holes on the brane.

On the other hand, Youm showed that the RS solution can be found in the dilatonic domain wall [11,12. In order to study physics on the brane, we need its effective action.

\footnotetext{
${ }^{1}$ In fact, this gauge for $h_{M N}$ is composed of Gaussian-Normal (GN) gauge $\left(h_{44}=h_{4 \mu}=0\right)$ and $4 \mathrm{D}$ transverse, tracefree (TTF) gauge $\left(\partial^{\mu} h_{\mu \nu}=0, h_{\mu}^{\mu}=0\right)$.

${ }^{2}$ This corresponds to the 5D TTF gauge $\left(\partial_{M} h^{M N}=0, h_{M}^{M}=0\right)$.

${ }^{3}$ Precisely, this is not a 5D black hole solution to the RS model. At present, nobody knows that.
} 
It is known that zero mode effective action takes a form of the Brans-Dicke model with a potential.

In this paper, we study stability of the large $\mathrm{RS}$ black hole with the $z$-independent perturbations such as $h_{\mu \nu}(x), h_{44}(x)$. We find that these are massless graviton and scalar modes propagating in the RS black hole background. Here we do not require the 4D TTF gauge which is useful for the RS Minkowski space. Instead we choose the Regge-Wheeler (RW) gauge for our study of spherically symmetric background.

\section{RANDALL-SUNDRUM SOLUTION AND BRANS-DICKE TYPE MODEL}

We start with the 5D bulk action and the 4D domain wall action as 12

$$
S=S_{\mathrm{bulk}}+S_{\mathrm{DW}}
$$

with

$$
\begin{aligned}
& S_{\text {bulk }}=\frac{1}{2 \kappa_{5}^{2}} \int d^{5} x \sqrt{-G}\left[R_{5}-\frac{4}{3} \partial_{M} D \partial^{M} D-e^{-2 a D} \Lambda\right], \\
& S_{D W}=-\sigma_{\text {DW }} \int d^{4} x \sqrt{-\gamma} e^{-a D},
\end{aligned}
$$

where $\sigma_{\mathrm{DW}}$ is the tension of the domain wall and $\gamma$ is the determinant of the induced metric $\gamma_{\mu \nu}=\partial_{\mu} X^{M} \partial_{\nu} X^{N} G_{M N}$ for the domain wall. Here $M, N=0,1,2,3,4\left(x^{4}=z\right)$ and $\mu, \nu=0,1,2,3\left(x^{\mu}=x\right)$. " $D$ " denotes the dilaton. We are interested in the second RS solution with

$$
\bar{G}_{M N}=H^{-2}(z) \eta_{M N}, \quad \bar{D}=0, \quad \Lambda=-12 k^{2}, \quad \sigma_{\mathrm{DW}}=6 k / \kappa_{5}^{2}, \quad a=0
$$

with $H=k|z|+1$ and $\eta_{M N}=\operatorname{diag}[-++++]$. Here overbar $\left(^{-}\right)$means the background value. In this paper we follow the MTW conventions [13].

In order to obtain a $4 \mathrm{D}$ effective action, we introduce the metric $\hat{G}_{M N}$ in $G_{M N}=$ $H^{-2}(z) \hat{G}_{M N}$ which satisfies $\partial_{z} \hat{G}_{M N}=0$. Explicitly, the line element is given by

$$
\begin{aligned}
d S_{5}^{2} & =G_{M N} d x^{M} d x^{N}=H^{-2} \hat{G}_{M N} d x^{M} d x^{N} \\
& =H^{-2}\left[g_{\mu \nu}(x) d x^{\mu} d x^{\nu}+\Phi^{2}(x) d z^{2}\right] .
\end{aligned}
$$

Off-diagonal elements are not allowed because if they exist, these violate the $Z_{2}$-symmetry argument?. At this stage we wish to remind the reader that $\Phi(x)$ has nothing to do with the radion which is necessary for stabilizing the distance between two branes in the first RS model [1]. This is because here we consider the second RS model [2]. Substituting (5) with

\footnotetext{
${ }^{4}$ With this ansatz, the dilatonic action Eq.(1) reduces to the second RS model exactly [2]

${ }^{5}$ One may introduce off-diagonal term of $2 A_{\mu}(x) d x^{\mu} d z$. But this is not invariant under the reflection of $z \rightarrow-z$ [1]. Thus we must have $A_{\mu}(x)=0$.
} 
$D=0$ into (11) and integrating it over $z$ lead to the Brans-Dicke type model with a potential |12]

$$
S_{\mathrm{RS}}=\frac{1}{2 \kappa_{4}^{2}} \int d^{4} x \sqrt{-g}\left[\Phi R+6 k^{2}\left(\Phi+\frac{1}{\Phi}-2\right)\right]
$$

with $\kappa_{4}^{2}=k \kappa_{5}^{2}$. This is our key action which is suitable for the study of physics defined on the brane. Also it can be derived from the RS model directly. It is emphasized that the first term $(\Phi R)$ is the BD term with $\omega=0$ [14]. The BD model with $\omega=0$ corresponds to the massless Kaluza-Klein model with $g_{\mu \nu}, g_{44}\left(\sim \Phi^{2}\right)$, and $g_{\mu 4}\left(\sim A_{\mu}\right)=0$ [. In this sense, we wish to call $\Phi$ as the BD scalar. The second term arises from the fact that the $5 \mathrm{D}$ spacetime is an $\mathrm{AdS}_{5}$ with a negative cosmological constant $\Lambda$ and a domain wall located at $z=0$. Equivalently, this means that the RS solution gives us a non-factorizable geometry with $H=k|z|+1$. Hence this term accounts for the feature of the RS type solution and it plays the role of an effective potential. ¿From now on we wish to separate the pure BD model $\left(\mathcal{L}_{\mathrm{BD}}=\Phi R\right)$ from the RS model $\left(\mathcal{L}_{\mathrm{RS}}=\Phi R+6 k^{2}(\Phi+1 / \Phi-2)\right)$ for comparison.

¿From (6) we derive the equations of motion $\left(\delta_{\Phi} S_{\mathrm{RS}}=0, \delta_{g_{\mu \nu}} S_{\mathrm{RS}}=0\right)$

$$
\begin{aligned}
& R+6 k^{2}\left(1-\frac{1}{\Phi^{2}}\right)=0, \\
& R_{\mu \nu}-\frac{1}{2} g_{\mu \nu} R=\frac{1}{\Phi}\left\{\nabla_{\mu} \nabla_{\nu} \Phi-g_{\mu \nu} \square \Phi+3 k^{2}\left(\Phi+\frac{1}{\Phi}-2\right) g_{\mu \nu}\right\} .
\end{aligned}
$$

Contracting Eq.(8) with $g^{\mu \nu}$ and using Eq.(17) leads to the RS scalar equation

$$
\square \Phi+2 k^{2}\left(-\Phi-\frac{3}{\Phi}+4\right)=0 .
$$

Also from Eq.(8), its contraction form, and (9) one finds the other Einstein equation

$$
R_{\mu \nu}=\frac{1}{\Phi}\left\{\nabla_{\mu} \nabla_{\nu} \Phi+2 k^{2}(-\Phi+1) g_{\mu \nu}\right\} .
$$

Here one finds a solution which satisfies all of equations (7)-(10) simultaneously as

$$
\bar{\Phi}=1, \quad \bar{R}=0, \quad \bar{R}_{\mu \nu}=0 .
$$

This means that the Ricci-flat condition of $\bar{R}_{\mu \nu}=0$ with $\bar{\Phi}=1$ describes the $4 \mathrm{D}$ vacuum configuration correctly.

As an example, we choose the Minkowski spacetime

$$
\bar{g}_{\mu \nu}=\eta_{\mu \nu}, \quad \bar{\Phi}=1 .
$$

To study the propagations on this background, we introduce the perturbation around the background (12) as

\footnotetext{
${ }^{6}$ This model is based on the action of $S_{\mathrm{KK}}=\frac{1}{\kappa_{5}^{2}} \int d^{5} x \sqrt{-G} R_{5}$ with a factorizable geometry of $H=1$.
} 


$$
g_{\mu \nu}=\eta_{\mu \nu}+h_{\mu \nu}, \Phi=\bar{\Phi}+\varphi\left(g_{44}=\bar{g}_{44}+h_{44}\right) .
$$

Then the linearized equations to (9) and (10) are found to be

$$
\begin{aligned}
& \partial^{2} \varphi+4 k^{2} \varphi=0, \\
& \delta R_{\mu \nu}(h)-\partial_{\mu} \partial_{\nu} \varphi+2 k^{2} \varphi \eta_{\mu \nu}=0
\end{aligned}
$$

with

$$
\delta R_{\mu \nu}=-\frac{1}{2}\left[\partial^{2} h_{\mu \nu}+\partial_{\nu} \partial_{\mu} h_{\rho}^{\rho}-\partial^{\rho} \partial_{\mu} h_{\nu \rho}-\partial^{\rho} \partial_{\nu} h_{\mu \rho}\right] .
$$

Under the 4D TTF gauge, one finds that $\delta R_{\mu \nu}=-\frac{1}{2} \partial^{2} h_{\mu \nu}$ and $\eta^{\mu \nu} \delta R_{\mu \nu}=-\frac{1}{2} \partial^{2} h^{\mu}{ }_{\mu}=0$. Contracting Eq.(15) with $\eta^{\mu \nu}$ leads to the other equation for $\varphi$

$$
\partial^{2} \varphi-8 k^{2} \varphi=0 .
$$

Eq.(14) allows a tachyonic solution because it has a negative potential term of $-4 k^{2}$. Fortunately we resolve this problem. We have two different equations (14) and (17) for the same field of $\varphi$. Hence we require $\varphi=0$ for consistency. This observation agrees with Refs. [6, 12]. Then Eq.(15) reduces to an equation for the massless graviton without a matter source on the brane

$$
\partial^{2} h_{\mu \nu}=0
$$

\section{SCHWARZSCHILD BLACK HOLE SOLUTIONS}

Introducing a spherically symmetric spacetime, one obtains the Schwarzschild black hole with $\bar{\Phi}=1$ in the domain wall approach [0] as

$$
\begin{aligned}
& \bar{g}_{\mu \nu}=\operatorname{diag}\left[-e^{\nu}, e^{-\nu}, r^{2}, r^{2} \sin ^{2} \theta\right], \\
& \bar{R}_{t r t r}=2 \frac{M}{r^{3}}, \quad \bar{R}_{t \theta t \theta}=-\frac{(r-2 M) M}{r^{2}}, \quad \bar{R}_{t \phi t \phi}=\sin ^{2} \theta \bar{R}_{t \theta t \theta}, \\
& \bar{R}_{r \theta r \theta}=\frac{M}{r-2 M}, \quad \bar{R}_{r \phi r \phi}=\sin ^{2} \theta \bar{R}_{r \theta r \theta}, \quad \bar{R}_{\theta \phi \theta \phi}=-2 M r \sin ^{2} \theta, \\
& \bar{R}_{\mu \nu}=0, \quad \bar{R}=0
\end{aligned}
$$

with

$$
e^{\nu}=1-\frac{2 M}{r} .
$$

Here $M=G_{N} M_{4}=\kappa_{4}^{2} M_{4} / 8 \pi=G_{5} k M_{4}$ with $G_{N}$ (4D Newtonian constant) and $G_{5}$ (5D Newtonian constant). $M_{4}$ is the mass of a large black hole with $M_{4}>1 /\left(2 k^{2} G_{5}\right)$. The BD and RS black hole solutions are permitted because the Schwarzschild solution comes from the Ricci-flat condition. The BD (RS) black holes denote the Schwarzschild black hole with 
$\mathcal{L}_{\mathrm{BD}}\left(\mathcal{L}_{\mathrm{RS}}\right)$, respectively. The $\mathrm{BD}$ black is introduced for reference. To study these black holes specifically, we introduce the perturbation

$$
g_{\mu \nu}=\bar{g}_{\mu \nu}+h_{\mu \nu}, \quad \Phi=\bar{\Phi}+\varphi .
$$

Then the linearized equations to Eqs. (9) and (10) are found as

$$
\begin{aligned}
& \dot{\square} \varphi+4 k^{2} \varphi=0, \\
& \delta R_{\mu \nu}(h)-\bar{\nabla}_{\nu} \bar{\nabla}_{\mu} \varphi+2 k^{2} \varphi \bar{g}_{\mu \nu}=0
\end{aligned}
$$

with the Lichnerowicz operator $\delta R_{\mu \nu}(h)$ [15, 16]

$$
\delta R_{\mu \nu}(h)=-\frac{1}{2}\left[\bar{\square} h_{\mu \nu}+\bar{\nabla}_{\nu} \bar{\nabla}_{\mu} h_{\rho}^{\rho}-\bar{\nabla}^{\rho} \bar{\nabla}_{\mu} h_{\nu \rho}-\bar{\nabla}^{\rho} \bar{\nabla}_{\nu} h_{\mu \rho}\right] .
$$

We note that Eq. (23) is not a diagonalized form to obtain eigenmodes. If one introduces $\hat{h}_{\rho \nu}=h_{\rho \nu}+\varphi \bar{g}_{\rho \nu}$, then this leads to

$$
\delta R_{\mu \nu}(\hat{h})=0 .
$$

This is the perturbed equation of pure $4 \mathrm{D}$ gravity for $\hat{h}_{\mu \nu}$ ?. A way to analyze Eqs.(22) and (23) is known 19,20]. For instance, it is possible if one uses the RW gauge instead of the 4D TTF gauge. The perturbation $\hat{h}_{\mu \nu}$ falls into two distinct classes - odd and even parities with $(-1)^{l+1}$ and $(-1)^{l}$, respectively. $l$ denotes an angular quantum number on $S^{2}: \bar{L}^{2} Y_{l m}(\theta, \phi)=$ $-l(l+1) Y_{l m}(\theta, \phi)$. Among ten components in the axisymmetric perturbation, one can always choose six components by taking into account the general coordinate transformations : $x^{\mu \prime}=$ $x^{\mu}+\epsilon \xi^{\mu}[21,22]$. This is a choice of the RW gauge. And this is obvious here because we consider the propagation of gravitons on the brane. In the RW gauge we assign two components $\left(h_{0}, h_{1}\right)$ for odd parity

$$
\hat{h}_{\mu \nu}^{\text {odd }}=\left(\begin{array}{cccc}
0 & 0 & 0 & h_{0}(r) \\
0 & 0 & 0 & h_{1}(r) \\
0 & 0 & 0 & 0 \\
h_{0}(r) & h_{1}(r) & 0 & 0
\end{array}\right) e^{-i \omega t} \sin \theta \frac{d P_{l}(\theta)}{d \theta}
$$

with Legendre polynomial $P_{l}(\theta)$. For even parity, we have $\hat{h}_{\mu \nu}^{e v e n}=h_{\mu \nu}^{e v e n}+\varphi \bar{g}_{\mu \nu}$, where $h_{\mu \nu}^{e v e n}$ is composed of four components $\left(H_{0}, H_{1}, H_{2}, K\right)$ as

$$
h_{\mu \nu}^{\text {even }}=\left(\begin{array}{cccc}
H_{0}\left(1-\frac{2 M}{r}\right) & H_{1} & 0 & 0 \\
H_{1} & H_{2}\left(1-\frac{2 M}{r}\right)^{-1} & 0 & 0 \\
0 & 0 & r^{2} K(r) & 0 \\
0 & 0 & 0 & r^{2} \sin ^{2} \theta K(r)
\end{array}\right) e^{-i \omega t} P_{l}(\theta) .
$$

These two cases are never mixed and thus they provide two degrees of freedom which is necessary for describing a massless spin-2 particle.

\footnotetext{
${ }^{7}$ Its stability analysis was performed 30 years ago [17,18.
} 


\section{A. RS scalar perturbation}

Let us first analyze the RS scalar perturbation using Eq.(22). Considering $\varphi \propto$ $\frac{\psi(r)}{r} Y_{l m}(\theta, \phi) e^{-i \omega t}$ and the background (19), one finds the Schrödinger-type equation

$$
\frac{d^{2} \psi}{d r^{* 2}}+\left(\omega^{2}-V_{\psi}^{\mathrm{RS}}\right) \psi=0
$$

where the RS scalar potential is given by

$$
V_{\psi}^{\mathrm{RS}}(r)=\left(1-\frac{2 M}{r}\right)\left\{\frac{l(l+1)}{r^{2}}+\frac{2 M}{r^{3}}-4 k^{2}\right\}
$$

with the tortoise coordinate $r^{*}=r+2 M \ln \left(\frac{r}{2 M}-1\right)$. ¿From the analysis in ref. [20], we find that for $\omega=i \alpha, 0<\alpha<2 k$, the scalar perturbation has an exponentially growing mode of $e^{\alpha t}$. Therefore this system may be classically unstable. In other words, " $-4 k^{2} "$ in the potential induces an instability of the asymptotically flat space of $r \rightarrow \infty$. Thus we call it as a potential instability. This may imply that the RS black hole solution is classically unstable. However, it is not true. Any exponentially growing mode is not allowed for the RS spherically symmetric background. An important point what we remind is that an $\mathrm{AdS}_{5}$ acts like a box of size $\mathrm{y}^{8} \sim(2 k)^{-1}$ [7]. So it may allow an unstable perturbation of wavelength with $\lambda \leq(2 k)^{-1}$ only [23]. However, for this case, we cannot find any consistent (unstable) solution [20]. In the unstable case of $0<\alpha=\lambda^{-1}<2 k$, one finds a condition of $\lambda>(2 k)^{-1}$ which is forbidden inside an $\mathrm{AdS}_{5}$. The instability problem can be cured by considering the bulk spacetime. Hence we can include the RS scalar mode, as a physical field, which propagates in the RS black hole background.

\section{B. Odd parity perturbation for $\hat{h}_{\mu \nu}$}

Now we discuss the odd parity perturbation for $\hat{h}_{\mu \nu}$. ¿From Eq.(23), we have three equations :

$$
\delta R_{03}=\delta R_{13}=\delta R_{23}=0
$$

Here we obtain the Regge-Wheeler equation using $Q \equiv \frac{h_{1}}{r}\left(1-\frac{2 M}{r}\right)$

$$
\frac{d^{2} Q}{d r^{* 2}}+\left(\omega^{2}-V_{\mathrm{RW}}(r)\right) Q=0
$$

where the Regge-Wheeler potential is given by

\footnotetext{
${ }^{8}$ Although a conventional length scale of an $\mathrm{AdS}_{5}$ is determined by $\int d^{5} x \sqrt{-G} R_{5}$ in Eq.(2) as $\int d z H^{-3}=1 / k$, we here choose a size of the $\mathrm{AdS}_{5}$-box as $1 /(2 k)$ for a definite calculation. This comes from : $-\Lambda \int d^{5} x \sqrt{-G} \rightarrow \int d z H^{-5}=1 /(2 k)$.
} 


$$
V_{\mathrm{RW}}(r)=\left(1-\frac{2 M}{r}\right)\left(\frac{l(l+1)}{r^{2}}-\frac{6 M}{r^{3}}\right) .
$$

Further $h_{0}$ is not an independent mode, it can be expressed in terms of $Q$ as $h_{0}=\frac{i}{\omega} \frac{d}{d r^{*}}(r Q)$. We note that the BD black hole [19] takes the same potential as in Eq.(32) for the odd parity perturbation. This case has already been analyzed by Vishveshwara in ref. [17] and an allowed solution is a scattering state

$$
\begin{aligned}
Q_{\infty} & =e^{-i \omega r^{*}}+A^{-} e^{i \omega r^{*}}\left(r^{*} \rightarrow \infty\right), \\
Q_{2 M} & =B^{-} e^{-i \omega r^{*}}\left(r^{*} \rightarrow-\infty\right) .
\end{aligned}
$$

\section{Even parity perturbation for $\hat{h}_{\mu \nu}$}

In this case, from the remaining seven equations, we have the Zerilli equation in ref. [17]

$$
\frac{d^{2} \psi_{\mathrm{Z}}}{d r^{* 2}}+\left(\omega^{2}-V_{\mathrm{Z}}(r)\right) \psi_{\mathrm{Z}}=0
$$

where the Zerilli potential is given by

$$
V_{\mathrm{Z}}(r)=\left(1-\frac{2 M}{r}\right)\left\{\frac{2 \lambda^{2}(\lambda+1) r^{3}+6 \lambda^{2} M r^{2}+18 \lambda M^{2} r+18 M^{3}}{r^{3}(\lambda r+3 M)^{2}}\right\}
$$

with $\lambda=(l-1)(l+2) / 2$. Here $\psi_{\mathrm{Z}}(r)$ is a gauge invariant combination of $H_{0}, H_{1}, H_{2}, K, \psi / r$ 19, 21,22]. At this stage we would like to comment that the BD equation $\left(\delta R_{\mu \nu}(h)-\right.$ $\left.\nabla_{\nu} \nabla_{\mu} \varphi=0\right)$ in ref. [19] takes the same equation as in Eq.(34). Also it is easily shown that an allowed solution is a plane wave like Eq.(33). This fact can be easily read off from the shape of potentials $V_{\mathrm{RW}}$ and $V_{\mathrm{Z}}$. Because these all belong to positive potential barrier for $l \geq 2$, there exist scattering states only. In other words, there are no bound state solutions. This means that one cannot find any exponentially growing mode in the graviton sector, even if $k^{2}$-term is involved in the RS equation (23).

\section{DISCUSSIONS}

We investigate the zero mode sector of the 5D dilatonic domain wall solution. This sector is very useful for describing the RS black hole on the brane. Assuming a spherically symmetric spacetime, one has a large black hole on the brane. We perform the analysis of stability to see whether or not the RS black hole truly exists. It is well-known that the BD black hole is stable. Here one finds an exponentially growing mode for the RS black hole because of a negative nature of its potential. If there is an exponentially growing mode, its black hole is classically unstable. ¿From the analysis of $\varphi$ in the RS Minkowski spacetime, we have $\varphi=0$ under the $4 \mathrm{D}$ TTF gauge. On the other hand, we cannot have $\varphi=0$ in the spherically symmetric black hole spacetime with the RW gauge. Fortunately, we find

that this instability is not allowed inside an $\mathrm{AdS}_{5}$ whose size is $(2 k)^{-1}$. Hence the instability 
problem is cured. Actually BD scalar as well as gravitons can propagate in the black hole spacetime. Also this implies that the large RS black hole is stable.

For the metric perturbations, we don't worry about their stability even for considering $\varphi \neq 0$. Choosing the RW gauge, the graviton sector including $\varphi$ leads to the well-known two classes of odd and even-parities. Since this sector has always positive potential barriers for any $l$ with $l \geq 2$ [18], there are no exponentially growing modes. Also this can be confirmed

from the other side. If we introduce a new tensor $\hat{h}_{\mu \nu}$, Eq. (23) reduces to Eq. (25). This is nothing but the perturbed equation for pure 4D gravity, which was proved to be stable thirty years ago.

In conclusion, the large black hole in the dilaton domain wall is stable. This can represent a stable form on the brane for the RS black cigar whose metric is not known up to now. Finally we comment that the RS black hole can be described by massless gravitons and a scalar mode with smaller wavelength than the size of an $\mathrm{AdS}_{5}$-box.

\section{ACKNOWLEDGEMENT}

We thank to J.Y. Kim and G. Kang for hepful discussions. This work was supported in part by the Brain Korea 21 Program of Ministry of Education, Project No. D-0025 and KOSEF, Project No. 2000-1-11200-001-3. 


\section{REFERENCES}

[1] L. Randall and R. Sundrum, Phys. Rev. Lett. 83 (1999)3370, hep-ph/9905221.

[2] L. Randall and R. Sundrum, Phys. Rev. Lett. 83 (1999)4690, hep-th/9906064.

[3] N. Arkani-Hamed, S. Dimopoulos, G. Dvali and N. Kaloper, hep-th/9907209; W.D. Goldberger and M.B. Wise, hep-th/9907447; E. Halyo, hep-th/9909127; A. Chamblin and G.W. Gibbons, hep-th/9909130.

[4] M.G. Ivanov and I.V. Volovich, "Metric Fluctuations in Brane Worlds", hep-th/9912242.

[5] Y.S. Myung and G. Kang, "Comment on "Metric Fluctuations in Brane Worlds"", hep-th/0001003.

[6] Y.S. Myung, G. Kang and H.W. Lee, "Randall-Sandrum gauge in brane world", hepth/0001107.

[7] A. Chamblin, S.W. Hawking and H.S. Reall, "Brane-world black holes", hepth/9909205.

[8] R. Gregory and R. Laflamme, Phys. Rev. Lett. 70 (1993) 2839; Nucl. Phys. B428 (1994) 399.

[9] R. Gregory, "Black string instabilities in anti-de Sitter space", hep-th/0004101.

[10] R. Emparan, hep-th/0009136.

[11] D. Youm, "Solutions in brane worlds II", hep-th/0001018.

[12] D. Youm, "A note on solutions in brane worlds", hep-th/0001166.

[13] C. Misner, K. Thorne, and J. Wheeler, Gravitation (Freeman and Company, San Francisco, 1973).

[14] C. Brans and R.M.D. Dicke, Phys. Rev. 124 (1961) 925; R.M.D. Dicke, Phys. Rev. 125 (1962) 2163.

[15] H.W. Lee, Y.S. Myung, and J.Y. Kim, Phys. Rev. D58 (1998) 104006, hep-th/9708099;

H.W. Lee, N.J. Kim, and Y.S. Myung, Class. Quantum Grav. 17 (2000) 113, hepth/9805050; H.W. Lee, N.J. Kim, Y.S. Myung, and J.Y. Kim, Phys. Rev. D57 (1998) 7361, hep-th/9801152.

[16] Y.S. Myung, G. Kang and H.W. Lee, "S-wave absorption of scalars by noncommutative D3-branes", hep-th/9912288.

[17] T. Regge and J. Wheeler, Phys. Rev. 108 (1957) 1403; C.V. Vishveshwara, Phys. Rev. D1 (1970) 2870; E.F. Zerilli, Phys. Rev. Lett. 24 (1970) 327.

[18] S. Chandrasekar, The Mathematical Theory of Black Holes ( Oxford Univ., New York, 1992).

[19] O. Kwon, Y. Kim, Y.S. Myung, B.H. Cho, and Y.J. Park, Phys. Rev. D34 (1986) 333.

[20] O. Kwon, Y. Kim, Y.S. Myung, B.H. Cho, and Y.J. Park, Int. J. Mod. Phys. A1 (1986) 709.

[21] A. Garat and R. Price, "Gauge invariant formalism for second order perturbations of the Schwarzschild spacetimes", gr-qc/9909005.

[22] C. Nicasio, R. Gleiser and J. Pullin, "Second order perturbations of a Schwarzschild black hole: inclusion of odd parity perturbations", gr-qc/0001021.

[23] A. Chamblin, C. Csaki, J. Erlich, and T. Hollowood, Phys. Rev. D64 (2000) 044012, hep-th/0002076. 